\title{
Triterpenos Holostánicos con Actividad Antifúngica Obtenidos del Pepino de Mar Holothuria floridana, Recolectado en la Bahía de Cispatá, Córdoba-Colombia
}

\author{
Gilmar G. Santafé*, Miguel S. Guzmán y Omar L. Torres. \\ Universidad de Córdoba, Departamento de Química, Laboratorio Química de los productos naturales, \\ Carrera 6 No. 76-103, Montería, Córdoba-Colombia (e-mail: gsantafe@correo.unicordoba.edu.co, \\ msegundoguzman@correo.unicordoba.edu.co,omart2365@gmail.com).
}

* Autor a quien debe ser dirigida la correspondencia

Recibido Jul. 22, 2013; Aceptado Sep. 16, 2013; Versión final recibida Oct. 4, 2013

\begin{abstract}
Resumen
Se reporta por primera vez la obtención en su forma libre de los compuestos holosta-22,25- epoxi-7,9-dien$3 \beta$-ol (compuesto 1) y holosta-22,25-epoxi-7,9-dien-17-3ß-diol (compuesto 2), aislados del pepino de mar Holothuria floridana, recolectado en la bahía de Cispatá en el Caribe Colombiano. Los pepinos de mar del género Holothuria son conocidos como fuente de triterpenos glicosidados biológicamente activos. Los compuestos 1 y 2 fueron aislados y purificados mediante procesos cromatográficos y caracterizados químicamente utilizando técnicas combinadas de cromatografía líquida acoplada a espectrometría de masas (QSTAR Elite LC/MS) además de experimentos de resonancia magnética nuclear de protón y carbono 13. A los compuestos obtenidos se les evaluó su actividad fungicida frente al hongo fitopatógeno Sclerotiniasclerotiorum, encontrándose resultados promisorios en el compuesto 2.
\end{abstract}

Palabras clave:pepino de mar, Holothuria floridana, triterpenosholostánicos, actividad fungicida, Bahía de Cispatá

\section{Holostan-type Triterpene with Antifungal Activity Obtained from the Sea Cucumber Holothuriafloridana, Collected in the Cispatá Bay, Córdoba-Colombia}

\begin{abstract}

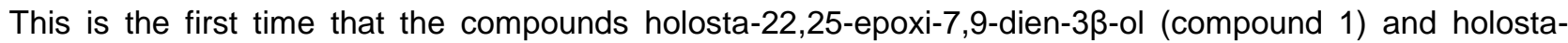
22,25-epoxi-7,9-dien-17-3ß-diol (compound 2) are obtained, in free form, from the sea cucumber Holothuriafloridana, collected in the Cispatá Bay in the Colombian Caribbean. Sea cucumbers of the Holothuria genus are known as sources of biologically active glycosylated triterpenes. Compounds 1 and 2 were isolated and purified via chromatographic processes and chemically characterized by using combined techniques of liquid chromatography coupled with mass spectrometry (QSTAR Elite LC/MS)in addition to proton and carbon 13 nuclear magnetic resonance experiments. The compounds obtained were evaluated for their fungicide activity against the phytopathogenic fungus Sclerotiniasclerotiorum, finding promising results in compound 2 .
\end{abstract}

Keywords: sea cucumber, Holothuria floridana, holostan-typetriterpenes, fungicideactivity, CispatáBay 


\section{INTRODUCCIÓN}

Los animales, las plantas y otros organismos que habitan los océanos del mundo, han constituido hasta el momento una rica e inagotable fuente de nuevos compuestos químicos. Esta tendencia se ha mantenido a partir del estudio de los diferentes y variados Phylum en los que se agrupan los seres marinos, uno de estos Phylum lo constituyen los equinodermos, de los cuales el número de nuevos metabolitos secundarios reportados anualmente ha sido relativamente constante durante la última década (Blunt, 2012).Las saponinas son uno de los tipos de compuestos químicos que se han logrado identificar en los equinodermos, en particular se han aislado de los pepinos de mar (Holothuroidea) y de las estrellas de mar (Asteroidea). Las Holothurinas, saponinas de holoturias que constituyen uno de los compuestos más representativos del género Holothuria, están formadas por carbohidratos y triterpenoides (Blunt, 2009), mientras que las asterosaponinas son básicamente glucósidos esteroidales. La mayoría de saponinas aisladas del género Holothuria han mostrado propiedades hemolíticas, antioxidantes (Althunibat, 2009), antitumorales, antiinflamatorias, antifúngicas (Ismail, 2008) ycitotóxicas (Shu-Yu, 2006; Yuan, 2008). Debido a la toxicidad general de las saponinas es posible considerar que estos compuestos actúen como agentes de defensa química. Las toxinas se concentran en órganos especializados conocidos como glándulas de Cuvier, que pueden ser exudadas para disuadir a los depredadores. Además de las saponinas, el género Holothuria presenta una diversidad de metabolitos tales como esteroles y ácidos grasos fosfolipídicos (Ismail, 2008).En su trabajo, Kitagawa y col (Blunt, 2009)identificaron a partir de la especie Holothuriahilla, el compuesto holothurina $B$, el cual mostró alta actividad antifúngica, también, se ha reportado el aislamiento e identificación química del compuesto Holothurina A3 como un triterpenoglicosidado con actividad citotóxica moderada, obtenido de Holothuriascabra (Blunt, 2009).

De otra parte, al extracto crudo y fracciones semipurificadas del pepino de mar mediterráneo Holothuriapolii, colectado en la bahía de Tabarka (costa de Túnez), se les evaluó su actividad antifúngica contra cepas de Aspergillus fumigatus, Trichophytonrubrum y Candidaalbicans utilizando el método de difusión en agar con variante de pozo. Los resultados obtenidos sugieren que las fracciones polares obtenidas a partir de los extractos acuosos y metanólicos podrían contener nuevos compuestos antifúngicos(Ismail, 2008).

Continuando con nuestras investigaciones sobre organismos marinos de la Bahía de Cispatá en el Caribe Colombiano, en este trabajo reportamos por primera vez la obtención en su forma libre, de los compuestos

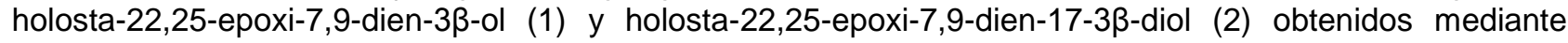
hidrólisis ácida de la fracción acuosa del pepino de mar Holothuria floridana. Los compuestos 1 y 2 fueron aislados y purificados mediante procesos cromatográficos e identificados químicamente utilizando técnicas combinadas de cromatografía líquida acoplada a espectrometría de masas (QSTAR Elite LC/MS), obteniéndose el valor de sus masas exactas, además de experimentos de resonancia magnética nuclear de protón y carbono 13. El compuesto 2 mostró buena actividad fungicida contra el hongo fitopatógeno Sclerotiniasclerotiorum.

\section{METODOLOGÍA}

Los especímenes del pepino de mar Holothuria floridana fueron recolectados en la Bahía de Cispatá, (09'22"N75'45"O), Departamento de Córdoba, Colombia,a una profundidad de un metro aproximadamente, posteriormente se congelaron hasta la preparación del material biológico para la obtención del extracto primario. La ubicación taxonómica del pepino de mar $\mathrm{H}$. floridana se llevó a cabo en el Departamento de Biología de la Universidad de Córdoba, Colombia, un espécimen se encuentra en el Laboratorio de Productos Naturales bajo el código PNM 024.El material recolectado (243 g) fue cortado en pedazos y sometido a percolación en $\mathrm{MeOH}$ por 3 días, luego se filtró y se destiló a presión reducida obteniéndose el extracto metanólico primario $(16.117 \mathrm{~g})$, el cual fue posteriormente sometido a fraccionamiento por reparto empleando solventes de polaridad creciente desde dicloro metano hasta agua. Ambas fases, la orgánica y la acuosa fueron concentradas de nuevo por destilación a presión reducida, obteniendo de la primera 5.943 $\mathrm{g}$ y de la segunda $9.787 \mathrm{~g}$.

Posteriormente, $4.527 \mathrm{~g}$ de la fase acuosa obtenida del pepino de mar $H$. floridana, fueron sometidos a hidrólisis ácida con $\mathrm{HCl} 2.5 \mathrm{~N}$ (relación muestra/ácido 1:6) (Benavides, 2012), en un equipo de reflujo con calentamiento y agitación continua durante tres horas. La suspensión del material hidrolizado fue neutralizada con bicarbonato de sodio, extraída con cloroformo y secada con sulfato de sodio anhidro, el extracto obtenido se concentró a sequedad bajo presión reducida y se monitoreó por cromatografía en capa delgada utilizando como referencia Colesterol (Sigma-Aldrich), obteniéndose $330 \mathrm{mg}$ de fracción hidrolizada.

La fracción obtenida $(330 \mathrm{mg}$ ) fue sometida a cromatografía en columna repetitiva sobre silica gel con un gradiente de elución desde bencina:AcOEt 5:1 hasta bencina:AcOEt 5:3, obteniéndose 4 subfracciones $\mathrm{HfH}_{1-4}$, las cuales se reunieron según su perfil en $\mathrm{CCD}$, la fracción $\mathrm{HfH}_{1}$ presentó igual perfil que el patrón 
utilizado. Esta fracción fue caracterizada portécnicas combinadas de Cromatografía de Gases de Alta Resolución acoplada a Espectrometría de masas de impacto electrónico (CGAR/EMIE). Para esto se utilizó una columna Agilent 122-0132 DB-1MS de longitud nominal $30 \mathrm{~m}$, diámetro $250 \mu \mathrm{m}$ y película de $0.25 \mu \mathrm{m}$, con una temperatura máxima de $340^{\circ} \mathrm{C}$, flujo constante de $1 \mathrm{~mL} / \mathrm{min}$, presión de 17.91 psi. Se utilizó un sistema de inyección splitless y helio como gas de arrastre, el volumen de inyección fue de $5 \mu \mathrm{L}$ con un tiempo de corrida de 65 minútos por muestra, fue utilizado un detector selectivo de masas con una fuente de ionización de $70 \mathrm{eV}$.

Las fracciónes $\mathrm{HfH}_{2}(10 \mathrm{mg})$ y $\mathrm{HfH}_{3}(6 \mathrm{mg})$, cuya apariencia física era de cristales blancos en forma de agujas, presentaron una sola mancha en CCD, estas dos fracciones fueron analizadas mediante técnicas combinadas de cromatografía líquida acoplada a espectrometría de masas empleando un equipo QSTAR Elite LC/MS,con una fuente de ionización Turbo Spray y un analizador de tiempo de vuelo (TOF), obteniéndose el valor de las masas exactas de los compuestos 1 y 2 . Se realizaron también experimentos de resonancia magnética nuclear de protón y carbono 13, utilizando un Espectrómetro de RMN Bruker de $300 \mathrm{MHz}$ modelo (AV300-SB), y como solvente cloroformo deuterado $\left(\mathrm{CDCl}_{3}\right)$, estos experimentos permitieron la asignación estructural de los compuestos 1 y 2 .

Para evaluar la actividad antifúngica de los compuestos aislados, se siguió el método de dilución en agar descrito por la NCCLS, previamente estandarizado (Marquez, 2007).Para el cultivo del hongo fitopatógeno Sclerotinia sclerotiorum se preparó un medio de cultivo PDA (Merck). Las cepas fueron suministradas por el laboratorio de Fitopatología de la Facultad de Ciencias Agrícolas de la Universidad de Córdoba. Las cajas de Petri y todos los materiales utilizados fueron esterilizados mediante autoclave. Se prepararon sensidiscos de papel (Whatman, № 3) de $7 \mathrm{~mm}$ de diámetro y se impregnaron con las soluciones de los compuestos preparadas en DMSO a 500 ppm, luego fue inoculado el hongo fitopatógeno Sclerotiniasclerotiorum, los sistemas se incubaron en condiciones aeróbicas a $32{ }^{\circ} \mathrm{C}$ con lecturas de zonas de inhibición a las $72 \mathrm{~h}$, las pruebas se realizaron por triplicado y se usaron como control positivo sensidiscos impregnados del fungicida comercial Colizym (Colinagro) (500 ppm).

\section{RESULTADOS Y DISCUSIÓN}

La hidrólisis de la fracción acuosa del pepino de mar $H$. floridana permitió la obtención de los compuestos triterpenoides holostánicos 1 y 2, los cuales fueron obtenidos como cristales blancos en forma de agujas.EI espectro de masas exactas (LC/MS TOF) del compuesto 1 mostró un ion seudomolecular $[\mathrm{M}+\mathrm{H}]^{+}$a $\mathrm{m} / \mathrm{z}$ : 469.3322, consistente con la fórmula molecular $\mathrm{C}_{30} \mathrm{H}_{45} \mathrm{O}_{4}{ }^{+}$cuyas masas exactas calculadas son de $\mathrm{m} / \mathrm{z}$ : 469.3312 (porcentaje de error de $2,1 \times 10^{-4} \%$ ). El espectro de RMN ${ }^{13} \mathrm{C}$ muestra señales para 30 carbonos corroborando la fórmula molecular propuesta inicialmente, entre las señales, se destacan las que resuenan a: $\delta 120.88,139.84,147.88$ y 111.75 ppm, las cuales corresponden a 4 carbonos alquénicos y la señal a: $\delta$ $177.47 \mathrm{ppm}$ perteneciente a un carbono carbonílico que se encuentra formando un anillo lactónico. El experimento DEPT 135, permitió identificar 7 carbonos metílicos, 8 carbonos metilénicos, 6 carbonos metínicos y 9 carbonos cuaternarios (tabla 1). Por comparación bibliográfica con datos espectroscópicos informados para compuestos aislados a partir del género Holothuria se logró establecer que las señales son características de triterpenos holostánicos (Shu-Yu, 2006; Yuan, 2008;Nguyen, 2006).El espectro de RMN ${ }^{1} \mathrm{H}$ muestra señales características a campo bajo como la señal a $85.55 \mathrm{ppm}$ (doblete ancho) que integra para un protón y la señal en $\delta 5.24$ ppm (multiplete), que integra para un protón, señales que son características de triterpenos holostánicos con insaturaciones en $\mathrm{H}-7$ y H-9(11). La señal en $\delta 4.06$ (t) que integra para un protón es característica de un protón en la posición C-22 de un anillo epoxi, este comportamiento permitió sugerir que el triterpeno no posee una cadena lateral abierta sino en forma de anillo, generando otra insaturación, comportamiento acorde con la fórmula molecular obtenida del espectro de masas. La señal en $\delta 3.25 \mathrm{dd}(\mathrm{J}=1.8$ y $4.2 \mathrm{~Hz})$ es originada por el protón con estereoquímica alfaen posición C-3, la cual aparece desplazada por la presencia de un oxígeno en este mismo carbono, la multiplicidad y las diferencias en las constantes de acoplamiento, son debidas a la presencia de dos protones diasterotópicos en el carbono adyacente, es decir, en C-2. El protón en C-17 resuena en $\delta 2.40$ y su multipilicidad $\mathrm{dd}(\mathrm{J}=3.01,10.55 \mathrm{~Hz})$, es consecuencia de la inequivalencia química de los hidrógenos adyacentes, los demás datos se muestran en la tabla 1.

Con base en el análisis espectroscópico y por comparación con los datos de la bibliografía especializada (Shu-Yu, 2006; Yuan, 2008;Nguyen, 2006;Reich, 2010)el compuesto 1 fue identificado como holosta-22,25epoxi-7,9-dien-3ß-ol, cuya estructura se muestra en la figura 1.

Por su parte el compuesto 2, está muy relacionado estructuralmente con el compuesto 1, presentando en RMN ${ }^{1} \mathrm{H}$ y ${ }^{13} \mathrm{C}$ un patrón de señales muy parecido, diferenciándose básicamente en dos aspectos, el primero tienen que ver con la señal que en el espectro de protónica para el compuesto 1 resuena en $\delta 2.40$, dd (3.01, 10.55) y que pertenece al protón $\mathrm{H}-17$, la cual no está presente en el compuesto 2 ;el segundo aspecto, se 
relaciona con la señal originada por los protones de C-16, que aparece en el compuesto 2 más desplazada $(\delta 2.35 \mathrm{~m}, 2 \mathrm{H})$ al compararla con la señal originada por los protones de este mismo carbono en el compuesto $1(\delta 1.66 \mathrm{~m}, 2 \mathrm{H})$, por lo tanto, para justificar este comportamiento es necesario ubicar un grupo hidroxilo en el carbono 17 del compuesto 2. Para confirmar la estructura y la presencia del grupo hidroxilo se analizó el espectro de masas exactas del compuesto 2, el cual presentó un ión seudomolecular $[\mathrm{M}+\mathrm{H}]^{+}$a $\mathrm{m} / \mathrm{z}$ : 485.3266 consistente con la fórmula molecular $\mathrm{C}_{30} \mathrm{H}_{45} \mathrm{O}_{5}{ }^{+}$, cuyas masas exactas calculadas corresponden a $\mathrm{m} / \mathrm{z}$ : 485.3262 (porcentaje de error: $2,1 \times 10^{-4} \%$ ), siendo mas pesado en 16 unidades que el compuesto 1 , ratificando la presencia del grupo $-\mathrm{OH}$. La fórmula molecular propuesta corresponde con un índice de deficiencia de hidrógeno (IDH) de 9. De acuerdo con lo anterior, el compuesto 2, fue identificado como holosta-22,25-epoxi-7,9-dien-17-3ß-diol (Fig. 2). Los demás datos espectroscópicos se muestran en la tabla 1.

Tabla 1. Datos de $\mathrm{RMN} 300 \mathrm{MHz}\left(\mathrm{CDCl}_{3}\right)$ para los compuestos 1 y 2.

\begin{tabular}{|c|c|c|c|c|c|c|}
\hline & \multicolumn{3}{|c|}{ Compuesto1 } & \multicolumn{3}{|c|}{ Compuesto2 } \\
\hline Atom & ${ }^{1} \mathrm{H} \delta / \mathrm{ppm}$ & ${ }^{13} \mathrm{C}$ & $\begin{array}{l}\text { DEPT } \\
135\end{array}$ & ${ }^{1} \mathrm{H} \delta / p p m$ & ${ }^{13} \mathrm{C}$ & DEPT 135 \\
\hline 1 & $1.39, \mathrm{~m}$ & 35.86 & $\mathrm{CH}_{2}$ & $1.40, \mathrm{~m}$ & 35.84 & $\mathrm{CH}_{2}$ \\
\hline 2 & $1.99, \mathrm{~m}$ & 27.20 & $\mathrm{CH}_{2}$ & $1.99, \mathrm{~m}$ & 27.20 & $\mathrm{CH}_{2}$ \\
\hline 3 & $3.25 \mathrm{dd}(1.8,4.2 \mathrm{~Hz})$ & 79.01 & $\mathrm{CH}$ & $\begin{array}{c}3.25 \mathrm{dd}(1.63,4.47 \\
\mathrm{Hz})\end{array}$ & 79.00 & $\mathrm{CH}$ \\
\hline 4 & --- & 38.75 & $\mathrm{C}$ & --- & 38.75 & $\mathrm{C}$ \\
\hline 5 & $1.06, \mathrm{~m}$ & 49.22 & $\mathrm{CH}$ & $1.05, \mathrm{~m}$ & 49.34 & $\mathrm{CH}$ \\
\hline 6 & $2.03, \mathrm{~m}$ & 23.02 & $\mathrm{CH}_{2}$ & 2.03, m & 23.02 & $\mathrm{CH}_{2}$ \\
\hline 7 & $5.55, \mathrm{brd}$ & 120.80 & $\mathrm{CH}$ & $5.52, \mathrm{brd}$ & 120.0 & $\mathrm{CH}$ \\
\hline 8 & --- & 139.84 & $\mathrm{C}$ & --- & 140.9 & $\mathrm{C}$ \\
\hline 9 & --- & 147.88 & C & --- & 147.3 & $\mathrm{C}$ \\
\hline 10 & --- & 37.59 & $\mathrm{C}$ & --- & 37.54 & $\mathrm{C}$ \\
\hline 11 & $5.24, \mathrm{~m}$ & 111.75 & $\mathrm{CH}$ & $5.26, m$ & 111.72 & $\mathrm{CH}$ \\
\hline 12 & $2.55, \mathrm{~m}$ & 27.77 & $\mathrm{CH}_{2}$ & $2.77, \mathrm{~m}$ & 27.77 & $\mathrm{CH}_{2}$ \\
\hline 13 & --- & 57.19 & $C$ & --- & 57.49 & $C$ \\
\hline 14 & --- & 48.22 & $\mathrm{C}$ & --- & 48.51 & $C$ \\
\hline 15 & $2.13, \mathrm{~m}$ & 23.48 & $\mathrm{CH}_{2}$ & 2.12, m & 28.43 & $\mathrm{CH}_{2}$ \\
\hline 16 & $1.66, \mathrm{~m}$ & 33.40 & $\mathrm{CH}_{2}$ & $2.35, \mathrm{~m}$ & 36.22 & $\mathrm{CH}_{2}$ \\
\hline 17 & $\begin{array}{c}2.40, \mathrm{dd}(3.01,10.55 \\
\mathrm{Hz})\end{array}$ & 51.66 & $\mathrm{CH}$ & --- & 86.44 & $\mathrm{C}$ \\
\hline 18 & --- & 177.47 & $\mathrm{C}$ & --- & 171.76 & $C$ \\
\hline 19 & $1.22, \mathrm{~s}$ & 26.03 & $\mathrm{CH}_{3}$ & $1.22, \mathrm{~s}$ & 25.03 & $\mathrm{CH}_{3}$ \\
\hline 20 & --- & 82.80 & $C$ & --- & 83.24 & $C$ \\
\hline 21 & $1.33, \mathrm{~s}$ & 22.45 & $\mathrm{CH}_{3}$ & $1.35, \mathrm{~s}$ & 22.45 & $\mathrm{CH}_{3}$ \\
\hline 22 & $4.06, \mathrm{t}$ & 79.87 & $\mathrm{CH}$ & $4.21, \mathrm{t}$ & 81.10 & $\mathrm{CH}$ \\
\hline 23 & $1.74, \mathrm{~m}$ & 33.52 & $\mathrm{CH}_{2}$ & $1.74, \mathrm{~m}$ & 33.57 & $\mathrm{CH}_{2}$ \\
\hline 24 & $1.71, \mathrm{~m}$ & 38.50 & $\mathrm{CH}_{2}$ & $1.71, \mathrm{~m}$ & 38.49 & $\mathrm{CH}_{2}$ \\
\hline 25 & --- & 81.31 & $C$ & --- & 81.63 & $C$ \\
\hline 26 & $1.10, \mathrm{~s}$ & 28.61 & $\mathrm{CH}_{3}$ & $1.10, \mathrm{~s}$ & 28.17 & $\mathrm{CH}_{3}$ \\
\hline 27 & $1.00, \mathrm{~s}$ & 27.47 & $\mathrm{CH}_{3}$ & $1.19, \mathrm{~s}$ & 26.03 & $\mathrm{CH}_{3}$ \\
\hline 28 & $0.90, \mathrm{~s}$ & 28.16 & $\mathrm{CH}_{3}$ & $0.90, \mathrm{~s}$ & 27.47 & $\mathrm{CH}_{3}$ \\
\hline 29 & $1.02, \mathrm{~s}$ & 15.81 & $\mathrm{CH}_{3}$ & $1.02, \mathrm{~s}$ & 15.81 & $\mathrm{CH}_{3}$ \\
\hline 30 & $1.26, \mathrm{~s}$ & 21.09 & $\mathrm{CH}_{3}$ & $1.25, \mathrm{~s}$ & 17.30 & $\mathrm{CH}_{3}$ \\
\hline
\end{tabular}


<smiles>CC1(C)CC[C@H]([C@]2(C)OC(=O)C34CC=C5C(=CCC6C(C)(C)[C@@H](O)CC[C@@]63C)[C@]4(C)CC[C@@H]52)O1</smiles>

Fig. 1: Holosta-22,25-epoxi-7,9-dien-3ß-ol (1)<smiles></smiles>

Fig. 2: Holosta-22,25-epoxi-7,9-dien-17-3ß-diol (2)

Este tipo de sustancias químicas ( 1 y 2 ) se encuentran normalmente formando compuestos del tipo saponinas, las cuales son utilizadas por los pepinos de mar como mecanismo de defensa. Los residuos triterpénicos de las holothurinas suelen contener el doble enlace $\Delta^{9(11)}$, lo que sugiere que estos compuestos son derivados de parkeol [lanost-9 (11)-en-3ß-ol]. Por su parte, los esteroles de pepinos de mar ya han sido caracterizados encontrándose que los principales compuestos de este tipo tienen un núcleo 14-metil $\Delta^{9(11)}$ y parecen estar relacionados metabólicamente (Kerr, 1995).

En estudios experimentales que utilizaron radio marcaje, se estudiaron las rutas biosintéticas de Holothuria mexicana, Holothuriaarenicola, y Stichopuscalifornicus, proponiéndose que todos seguían la ruta en la cual el escualeno, se cicla hasta parkeol o lanosterol con posterior demetilaciónformando los correspondientes esteroles (figura 3) (Djerassi, 1990).

En esta investigación realizada sobre el pepino de mar Holothuria floridana, se dedujo de los cromatogramas y espectros de la fracción $\mathrm{HfH}_{1}$ aislada a partir de la fase orgánica y de la obtenida de la hidrólisis de la fase acuosa, que no se encontraron compuestos tipo esterólicos, por lo tanto $H$. floridanadebe seguir la ruta biosintética que permite la formación de holothurinas con un anillo lactónico.

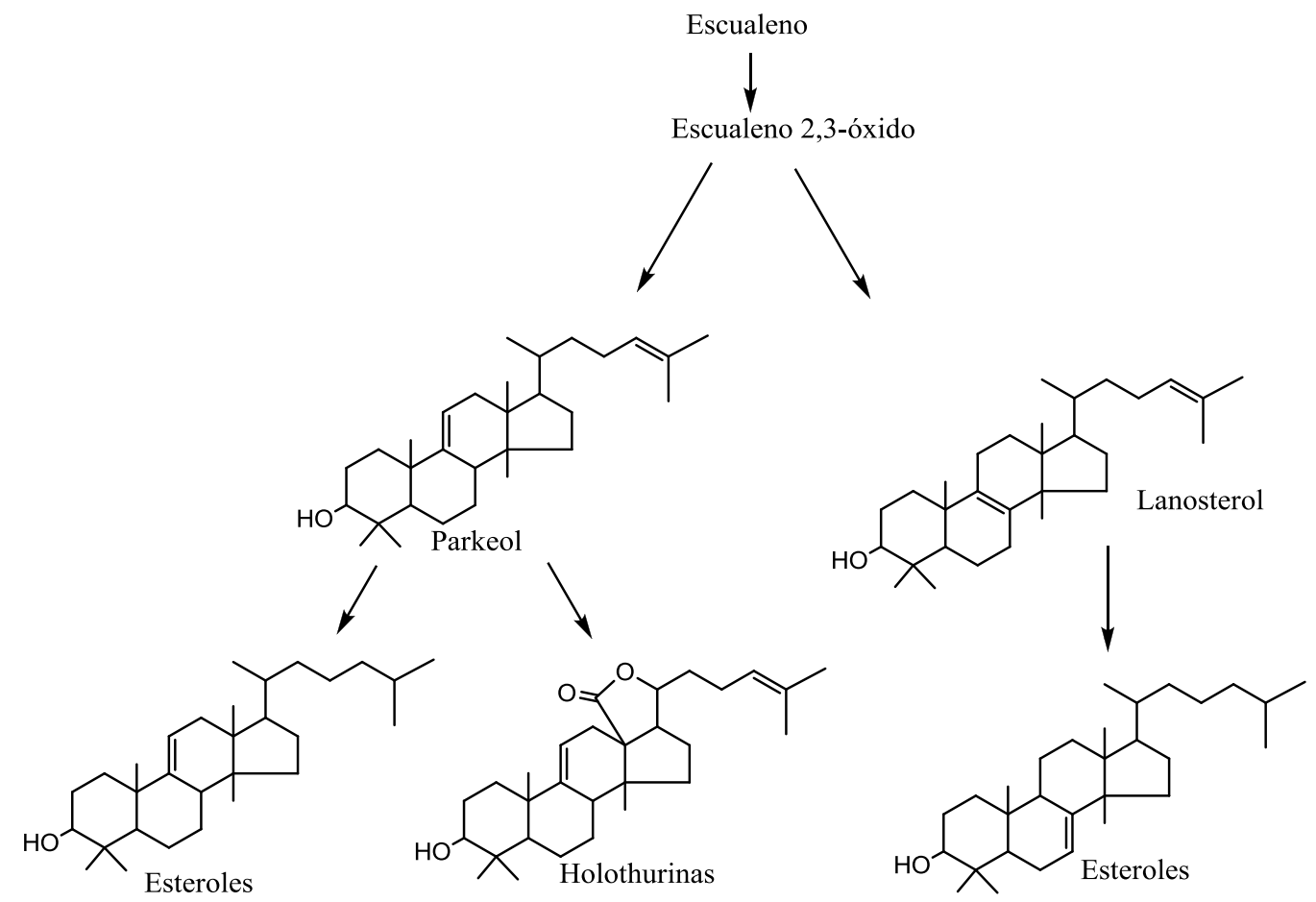

Fig.3: Esquema para la formación de esteroles y saponinas en pepinos de mar. 
En lo referente a la evaluación de la actividad antifúngica, se encontró que el control positivo (fungicida comercial Colizym (B) no permitió el crecimiento del hongo inhibiendo el 99\% del cultivo, mientras que el control negativo (DMSO) no presentó zona de inhibición, confirmando que el solvente utilizado en la preparación de las soluciones no afecta el crecimiento fúngico. Por su parte, el compuesto 1 no presentó zona de inhibición, mientras que el compuesto 2presentó un porcentaje de zona de inhibición del 93,98 \%, la cual se considera promisoria al compararla con el control positivo a la misma concentración (500 ppm). Estos resultados están de acuerdo con los reportados para pepinos de mar del género Holothuria, los cuales presentan considerable actividad antifúngica pero en la forma de triterpenos glicosidados (Blunt, 2009; Han, et., al. 2009), sin embargo, es importante destacar que en esta investigación se reportan en su forma libre, es decir, sin la parte glicósida. Comparando las dos estructuras, el compuesto 2 solo se diferencia del compuesto 1 en un sustituyente hidroxilo en la posición 16, por lo tanto, se deduce, que es este grupo hidroxilo el que le confiere la actividad antifúngica al compuesto 2.

\section{CONCLUSIONES}

A partir de la hidrolisis ácida de la fracción acuosa obtenida del pepino de mar Holothuria floridana se aislaron dos compuestos de tipo triterpenoide holostánico: Holosta-22,25-epoxi-7,9-dien-3ß-ol (1) y holosta22,25-epoxi-7,9-dien-17-3ß-diol (2), los cuales son reportados por primera vez en su forma libre, en este organismo. A estos compuestos se les evaluó su actividad fungicida contra el hongo fitopatógeno $S$. sclerotiurum, obteniéndose resultados promisorios para el compuesto 2. En esta investigación no se encontraron compuestos tipo esterólicos, por lo tanto se propone que este organismo marino sigue la ruta biosintética de formación de holothurinas.

\section{AGRADECIMIENTOS}

Los autores expresan su agradecimiento a la Oficina de Investigaciones de la Universidad de Córdoba, Colombia, por la financiación de este trabajo.

\section{REFERENCIAS}

Althunibat, O. y R. Hashim, In Vitro Antioxidant and Antiproliferative Activities of Three Malaysian Sea Cucumber Species, European Journal of Scientific Research,37, 376-387 (2009).

Benavides, O., O. Arango, A. Hurtado y M. Rojas, Cuantificación de Sapogeninas del Jugo Fresco y Fermentado de Fique (Furcraeagigantea) mediante Cromatografía Liquida de Alta Resolución (HPLC-PDA), Información Tecnológica, 23(3), 67-76 (2012).

Blunt, J. y B. Copp, Review: Marine natural products, Nat. Prod. Rep.,28, 196 (2012).

Blunt, J. y B. Copp, Review: Marine natural products, Nat. Prod. Rep.,26, 170-244 (2009).

Djerassi, C. y C. Lucinda, Biosynthetic Studies of Marine Lipids. Biosynthesis of and $\Delta 9(11)$-Sterols and Saponins in Sea Cucumbers, J. Org. Chem.,55, 2806-2813 (1990).

Han, H.,Y. YH, L. Li, B. Liu, M. La y H. Zhang. Antifungal active triterpene glycosides from sea cucumber Holothuriascabra, Yao Xue Xue Bao., 44(6), 620-624 (2009).

Ismail, H. y S. Lemriss, Antifungal activity of aqueous and methanolic extracts from the Mediterranean sea cucumber, Holothuriapolii, Journal de Mycologie Médicale, 18, 23-26 (2008).

Kerr, R. y Z. Chen, In vivo and in vitro biosynthesis of saponins in sea cucumbers, J. Nat. Prod.,58, 172-176 (1995).

Marquez, R. y C. De la Rosa, Actividad antifúngica del extracto total en etanol de la hojas frescas de Pedilanthus tithymaloides (ultimorrial), Scientia et technica año XIII, 33, 155-159 (2007).

Nguyen, $\mathrm{V}$ y N. Dang, A new triterpene glycoside from the sea Cucumber Holothuriascabra collected In Vietnam, AJSTD,23(4), 253-259 (2006).

Reich, M. The oldestsynallactid sea cucumber (Echinodermata: Holothuroidea: Aspidochirotida), Palaontol Z, 84, 541-546 (2010).

Shu-Yu, Z. Y Yang-Hua, Bioactive Triterpene Glycosides from the Sea Cucumber Holothuriafuscocinerea, J. Nat. Prod.,69, 1492-1495 (2006).

Yuan, W. y H. Yi, Two Antifungal Active Triterpene Glycosides from Sea Cucumber Holothuria (Microthele) axiloga, Chinese Journal of Natural Medicines,6(2), 105-108 (2008). 